\title{
Adaptive optics assisted and optical coherence tomography guided fs-laser system for ophthalmic surgery in the posterior eye
}

Ben Matthias

Miroslav Zabic

Dorothee Brockmann

Alexander Krüger

Tammo Ripken 


\title{
Adaptive optics assisted and optical coherence tomography guided fs-laser system for ophthalmic surgery in the posterior eye
}

\author{
Ben Matthias, ${ }^{\mathrm{a}, *}$ Miroslav Zabic, ${ }^{\mathrm{a}}$ Dorothee Brockmann, ${ }^{\mathrm{b}}$ Alexander Krüger, ${ }^{\mathrm{a}}$ and Tammo Ripken ${ }^{\mathrm{a}}$ \\ aLaser Zentrum Hannover e.V., Biomedical Optics Department, Hollerithallee 8, 30419 Hannover, Germany \\ ${ }^{\mathrm{b} H a n n o v e r ~ M e d i c a l ~ S c h o o l, ~ C l i n i c ~ f o r ~ O p h t h a l m o l o g y, ~ C a r l-N e u b e r g-S t r a ß e ~ 1, ~} 30625$ Hannover, Germany
}

\begin{abstract}
While fs-lasers are clinically established for surgery in the anterior eye, their use in the posterior eye is impeded by aberrations and focus position errors. We implemented a laboratory system to investigate whether fs-laser surgery in the posterior eye is made more feasible by aberration correction and tomographic image guidance. Aberration correction is obtained by adaptive optics (AO) and the image guidance is accomplished by optical coherence tomography (OCT). System characteristic measurements and cutting experiments were performed inside an eye model. By aberration correction, wavefront errors were reduced from $270 \mathrm{~nm}$ rootmean-square $(\mathrm{rms})$ to $64 \mathrm{~nm} \mathrm{rms}$, ignoring Zernike terms for tilts and focus. The Strehl ratio of the assigned point spread function is improved from 0.11 to 0.78 . The threshold pulse energy of laser-induced optical breakdown in water is lowered from about 3.0 to about $1.3 \mu \mathrm{J}$ measured at the eye model entrance. After laser cutting of a synthetic foil placed $300 \mu \mathrm{m}$ in front of porcine retinal tissue with the corrected system, postoperative threedimensional OCT imaging showed no lesions in the tissue. Our results corroborate that $A O$ and OCT will be two essential assistive components for possible clinical systems for fs-laser-based surgery in the posterior eye. (C) 2016 Society of Photo-Optical Instrumentation Engineers (SPIE) [DOI: 10.1117/1.JBO.21.12.121512]
\end{abstract}

Keywords: ophthalmic laser surgery; posterior eye segment; fs-laser; adaptive optics; aberrations; optical coherence tomography. Paper 160358SSPRR received Jun. 1, 2016; accepted for publication Nov. 17, 2016; published online Dec. $14,2016$.

\section{Introduction}

Fs-laser systems are an established part of ophthalmic surgery for minimally invasive dissection of corneal or crystalline lens tissue. This clinical application is very popular for corneal flap cutting during femtoLASIK (laser in situ keratomileusis). ${ }^{1,2}$ In lens surgery, fs-lasers have potential in the treatment of presbyopia (age-related reduction of accommodation amplitude) through lentotomy $y^{3,4}$ and are increasingly used in assisting cataract surgery. ${ }^{5,6}$ With $\sim 19$ million operations each year, cataract surgery is the most commonly performed surgical procedure worldwide $^{6}$ and offers a large market for commercial fs-laser systems. Because of the success of fs-laser surgery in the anterior eye, the consequential step is to open up new application possibilities for fs-lasers in the posterior eye. Possible applications include the retina and vitreoretinal and vitreal pathologies. For example, in the transition area between retina and vitreous, the treatment of tractive vitreoretinopathies is conceivable. Vitreomacular traction is defined by an atypical posterior hyaloid detachment with a persisting focal vitreoretinal adhesion to the fovea. This adhesion may be focal or broad, or there may be additional epiretinal membranes surrounding the adhesion. The adhesion may lead to a macular thickening, macular edema, and eventually a loss of visual acuity. Modern methods of examination, such as spectral domain optical coherence tomography (OCT), allow a detailed insight and analysis of the microretinal structures and of the vitreoretinal junction. Thus, the effect of vitreomacular traction can be very well documented and evaluated. ${ }^{7-10}$ In case of the completion of the posterior hyaloid

*Address all correspondence to: Ben Matthias, E-mail: b.matthias@Izh.de detachment, a spontaneous healing may occur. ${ }^{11}$ Currently, most cases of vitreomacular traction are treated by a surgical approach. The goal of vitrectomy is to relieve the traction. Surgery is usually directed at separating the posterior hyaloid. This usually requires stripping the posterior cortical vitreous from the retina and the peeling of epiretinal membranes. The ease of dissection is determined to a great extent by the type of the vitreoretinal adhesion, the extent of posterior vitreous separation, the presence of epiretinal membranes, and the degree of fibrosis of the adhesions. If traction can be relieved, the retinal swelling and edema usually resolve. The visual and anatomic results of this procedure have been good. Since a surgical approach may cause several complications, such as iatrogenic retinal holes, aspiration of the retina, bleeding, cataract induction, hypotony, and endophthalmitis, ${ }^{12}$ the intention of the laser approach is to relieve traction without opening the eyeball. We propose to relieve the traction via opening the posterior vitreous membrane circumscript to the adhesion. Figure 1, which shows an actual OCT image of a pathological eye and the intended locations of the laser cuts, illustrates our proposed method. We are aware that the adhesion of the central posterior vitreous membrane to the macula may continue, but since traction is removed, the macula should be able to recover. A possible leakage of vitreous fluid into the subhyaloidal space seems acceptable, although we have not yet evaluated the effects clinically. An opening of the posterior hyaloid membrane via $\mathrm{Nd}$ : YAG-laser has been published to be a safe therapeutical option in subhyaloidal hemorrhage. ${ }^{13,14}$

The effect exploited for fs-laser cutting inside a transparent medium is the laser-induced optical breakdown (LIOB) 


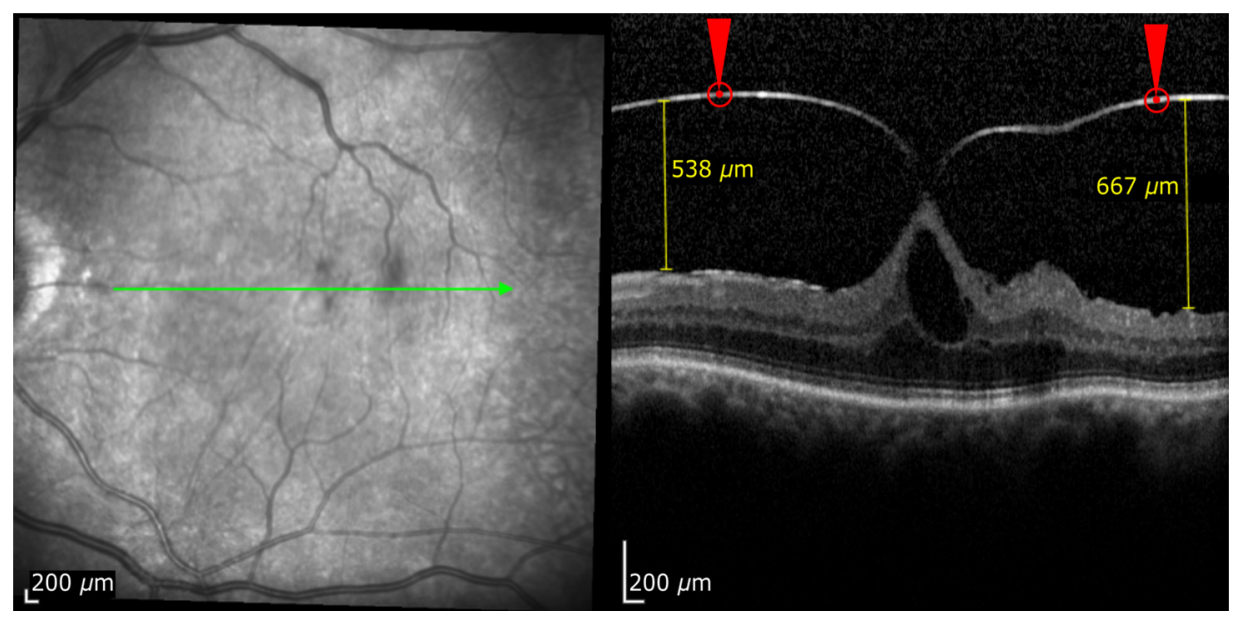

Fig. 1 Infrared reflectance photograph and OCT scan (Spectralis, Heidelberg Engineering, Germany) of a pathological eye showing a tractive membrane pulling on the retina. The arrows in the OCT scan indicate locations at which laser incisions in the direction of the image plane are intended to provide a relief of vitreoretinal traction.

mediated photodisruption of the tissue. As a consequence of focusing ultrashort laser pulses inside transparent media, such high irradiances (up to $\left.10^{11} \mathrm{~W} / \mathrm{cm}^{2}\right)^{15}$ can be achieved within the focal volume so that a plasma is generated by means of nonlinear ionization. If the density of free electrons exceeds a critical density of about $10^{21} \mathrm{~cm}^{-3},{ }^{16}$ the process is accompanied by mechanical effects and the surrounding material is disrupted locally. A sequence of these individual effects by scanning the laser focus within the medium allows the generation of a three-dimensional (3-D) cutting pattern. ${ }^{17}$

The irradiance is not only dependent on the irradiation time and pulse energy, but also on the irradiated area. A perfect optical system or a perfect eye would focus the incident light to a diffraction limited spot on the retina. However, in the case of a real system or a typical eye, aberrations have a noticeable impact and distort the laser focus. Aberrations can originate from numerous sources beginning at the laser system or the optical pulse delivery system including the focusing optics to the optics of the eye itself. While system aberrations can already be minimized to a large extent by careful optical design and alignment, aberrations of human eyes can be quite large. ${ }^{18}$ These aberrations primarily originate from the light passage through distorting ocular media, especially the cornea and the crystalline lens. Additional aberrations can be introduced depending on the application. An oblique passage of the laser beam through the ocular media during intraocular microsurgery, e.g., can contribute to the overall aberrations. ${ }^{19}$ Distortions of the laser focus reduce the precision and also raise the required pulse energy for cutting. The risk for peripheral implications and retinal damage is increased. Because target structures may be close to the retina, cutting precision and retinal safety are even more critical than in the established applications in the anterior eye. Consequently, for clinical laser applications in the posterior eye, it is of particular importance that aberrations are minimized.

Adaptive optics (AO) can provide aberration correction. A wavefront sensor [e.g., Shack-Hartmann sensor (SHS)] measures the system aberrations and the individual eye aberrations, and a wavefront modulator (e.g., deformable mirror) corrects for them. By AO aberration correction, the aberrated point spread function (PSF) of the incident light is reshaped toward the diffraction limit as has been shown for imaging devices such as fundus camera, ${ }^{20}$ scanning laser ophthalmoscope, ${ }^{21}$ and OCT. ${ }^{22}$
In addition, the threshold energy for fs-LIOB in water can be reduced $^{23}$ and the cutting precision can be enhanced ${ }^{24}$ by aberration correction with $\mathrm{AO}$.

Our presented laboratory system for image-guided vitreoretinal fs-laser surgery utilizes AO for spatial beam shaping and integrates OCT for assessing the vitreoretinal transition, the vitreoretinal traction, and the morphology of the fovea after vitreous detachment, and for guiding laser surgery based on this assessment. OCT already demonstrated its potential advantages for intrasurgical imaging and instrument guidance during conventional vitrectomy and membrane peeling. ${ }^{25-27}$ In the case of vitreomacular traction syndrome, the information obtained by means of OCT far exceeds the assessment of the intraoperative situation possible with the funduscopic image. For example, an exact statement about the locations of attachment of the posterior vitreous limiting membrane is possible and the preoperative OCT findings assist in planning the intraoperative procedure. ${ }^{28}$ Finally, OCT provides a postoperative control of success of vitreoretinal surgery. In fs-laser-based vitreoretinal surgical procedures, the laser focus must be accurately positioned at target structures while staying off vulnerable and highly absorbing retinal layers. OCT can provide qualitative and quantitative information, in particular in the axial direction, which is important to assist in surgical decision making where laser cuts must be avoided and where laser cuts should be applied in the epiretinal membrane. Before going into humans, thorough experiments are required which demonstrate the feasibility of image-guided AO assisted fs-laser surgery in the posterior eye. It is not the intent of this publication to provide a safe working distance to the retina. This important point will be the subject of a future study. Here, the developed laboratory system is presented in detail, the capability of the AO subsystem is demonstrated, and the laboratory system is used to perform OCT guided and $\mathrm{AO}$ enhanced fs-laser cutting of a phantom structure, which mimics the situation of an epiretinal membrane.

\section{Materials and Methods}

\subsection{System Overview}

Figure 2 shows a schematic overview of the optical system. The cutting laser is a Ti:sapphire fs-laser system (Spitfire: amplifier, 


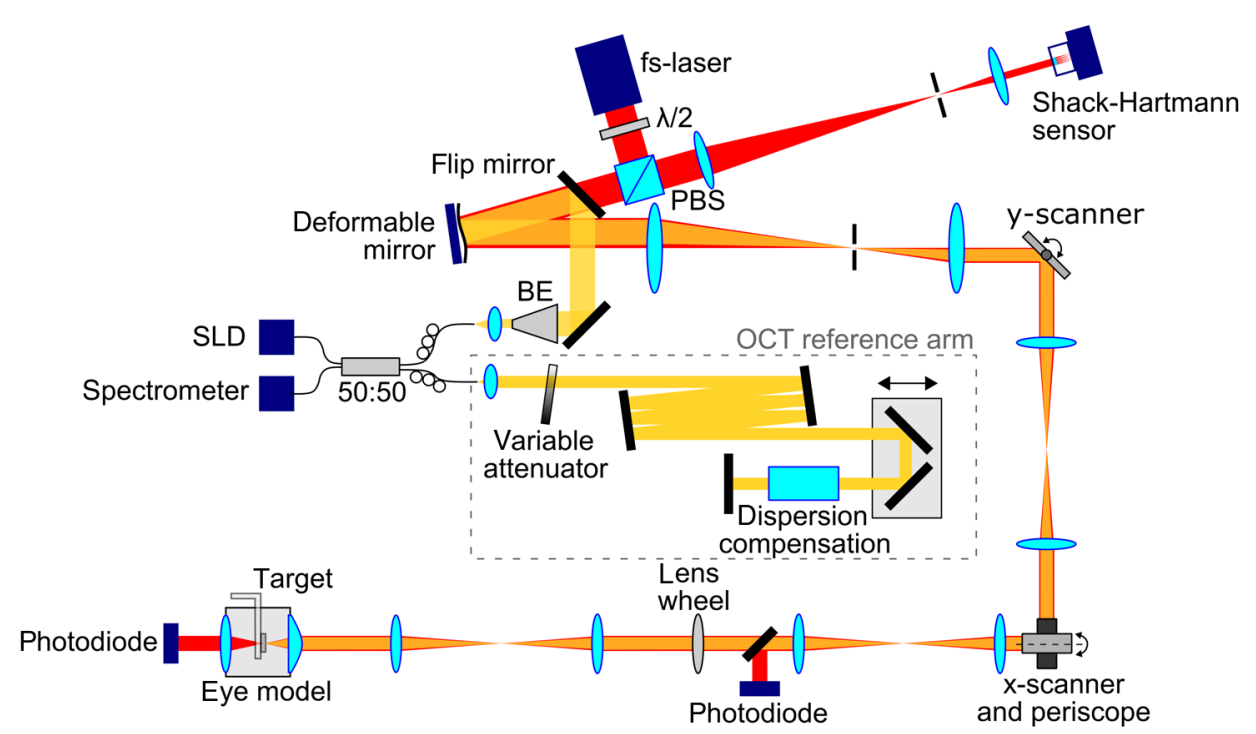

Fig. 2 Schematic overview of an AO assisted fs-laser system complemented by spectrometer-based Fourier domain OCT for image-guided cutting in an eye model. PBS, polarizing beam splitter; $\lambda / 2$, half-wave plate; BE, beam expander; and SLD, superluminescent diode.

MaiTai: seed, Empower: pump, Spectra-Physics, California) operated at a central wavelength of $800 \mathrm{~nm}$ and a repetition rate of $5 \mathrm{kHz}$. The measured pulse width was $250 \mathrm{fs}$ at the laser head output. The pulse energy in the setup is controlled by a half-wave plate and a polarizing beam splitter (PBS) within the lower microjoule range. The probe beam of the spectrometer-based Fourier domain OCT system is coupled into the same optical path as the fs-laser beam using a flip mirror. Lens-based telescopes relay the beams on the wavefront modulator, the $y$ and $x$ galvanometer scanning mirrors, through a lens wheel for adaptation of vergence, and onto the eye model. All telescopes following the first galvanometer scanning mirror are in $4 f^{\prime}$ configuration with customized lenses designed for diffraction limited quality up to $\pm 3.5 \mathrm{deg}$ optical scan angle.

\subsection{Adaptive Optics}

An SHS (HASO3-first, Imagine Eyes, France) is used for aberration measurement and a deformable mirror (DM) (mirao 52-e, Imagine Eyes, France) for aberration correction. The SHS, the $\mathrm{DM}$, the galvanometer scanning mirrors, the lens wheel, and the entrance lens of the eye model are placed in conjugated planes along the beam path. The correction amplitudes of the DM actuators are determined by analyzing the wavefront slopes measured by the SHS. For this purpose, an iterative closed loop algorithm of the analysis and control software Casao (Imagine Eyes, France) minimizes the root-mean-square (rms) value of the wavefront aberrations. The measuring signal is a point source produced by focusing fs-laser pulses at low energy levels on a diffuse reflecting target [band made of polytetrafluoroethylene (PTFE)] within the eye model. The beam originating at this point passes the system in reverse and is directed to the SHS. In order to prevent unwanted specular reflections from optical interfaces overlaying the measuring signal during the wavefront measurement, the first telescope following the DM is in off-axis configuration and a confocal pinhole in the telescope blocks reflections originating in the rear part of the setup. During fs-laser cutting and OCT imaging, the AO system is used in open loop operation. In this mode, the mirror surface of the DM is held in its corrected shape previously determined by closed loop correction.

Wavefront error maps, Zernike polynomial decompositions, and PSFs were determined with the software HASO 3.0 (Imagine Eyes, France) extended by an additional software package (PSF module, Imagine Eyes, France). The wavefront error maps were reconstructed with modal reconstruction using 32 Zernike coefficients and rms normalization. The zonal residual is added to the modal reconstruction. The Zernike rms polynomials are as ordered as by Wyant and Creath. ${ }^{29}$

\subsection{Optical Coherence Tomography}

The light of a fiber coupled broadband superluminescent diode (Broadlighter D 890-HP, Superlum, Ireland) with a 897.2-nm central wavelength and 151.7-nm spectral width enters a fiber coupler with a splitting ratio of 50:50. The reference arm of the OCT interferometer consists of a variable neutral density filter to adjust the reference light intensity, a folding mirror configuration, a moveable deflection unit to adjust for path length differences, dispersion compensation glasses, and a reflecting end mirror. The dispersion compensation glasses balance out most of the dispersion introduced by the optical elements in the sample arm. Residual dispersion imbalance is corrected numerically similar to a method as described by Köttig et al. ${ }^{30}$ Sample and reference arm fibers are looped through polarization control paddles and the polarization is adjusted for maximum contrast in the interference signal. The detection arm consists of a fiber coupled spectrometer (Custom Cobra Spectrometer with e2v EV71YEM4CL2010-BA8 camera, Wasatch Photonics, Utah) which provides an axial resolution (FWHM) of $3 \mu \mathrm{m}$ in air ( $2.3 \mu \mathrm{m}$ in water). The spectral interference fringes are captured by a 12-bit line scan camera with 2048 pixels in the wavelength range from 800 to $1030 \mathrm{~nm}$ giving a spectral resolution of $0.11 \mathrm{~nm} /$ pixel. The camera data are read by a high-speed camera link frame grabber (PCIe-1433, National Instruments, Texas) which serves as the master device for triggering the scanners. The two galvanometer scanning mirrors (GVS011/M, Thorlabs, New Jersey) and the telescopes direct and focus the OCT beam 
within the eye model. A simple line scan of the beam produces a brightness mode (B-mode) image. A series of line scans with a stepwise perpendicular offset between the lines produce a tomographic volume scan.

The acquisition and processing software is developed under Microsoft Visual C++ environment. Real-time image processing is performed in parallel on a graphics processing unit (GeForce GTX 660, NVIDIA Corporation, California) with NVIDIA's compute unified device architecture technology. The used platform is Windows 7 Professional, 64 bit on an Intel ${ }^{\circledR}$ Core $^{\mathrm{TM}}$ i7-3770T CPU, $2.50 \mathrm{GHz}$ with 16 GB RAM. As a first step of signal processing, the background signal is determined from the acquired raw frame by averaging a certain number of single lines in the frame. Autocorrelation terms and fixed pattern noise are removed via background subtraction. Afterward, the resulting spectra are recalibrated to be equally spaced in wavenumber and multiplied with the residual dispersive phase data determined by a preceding calibration routine. Then the signal is Hanning windowed for spectral shaping, Fourier transformed, logarithmized, and converted into gray scale values. Highly scattering structures in the eye model are imaged as bright pixels. The imaging speed (including the processing described above) for a $512 \times 1024$ pixels B-mode image is $31.6 \mathrm{fps}$ for the chosen acquisition parameters of $50 \mu$ s line integration time, $55 \mu$ s line period, and 64 additional line scan acquisitions for which the fast scanning axis is moved back to its start position.

\subsection{Eye Model}

The eye model consists of a water filled chamber with two opposite lenses for beam entrance and exit. The entrance lens is an uncoated aspheric condenser lens with an effective focus length of $17.0 \mathrm{~mm}$ in air and models the refractive power of the human eye. An incident collimated beam with a diameter of about $7.5 \mathrm{~mm}$ yields a numerical aperture of about 0.22 . For comparison, the maximum physiological pupil size and numerical aperture of the human eye are about $8 \mathrm{~mm}$ and $0.23 .^{31}$ The exit lens is a planoconvex lens with an effective focal length of $25.4 \mathrm{~mm}$ and collects the light for transmission measurements.

\subsection{Laser-Induced Optical Breakdown Threshold Energy Determination}

The LIOB threshold energy in water was determined by transmission measurements. For this purpose, the energy values at two energy calibrated photodiodes (PD10-V2 with Pulsar-2, Ophir Optronics, Israel) were compared (refer to Fig. 2). At each measuring point, the per pulse transmission of a given number of consecutive pulses was measured. Between each measuring point, the half-wave plate was rotated by a motorized rotation stage increasing the incident pulse energy. The measurement procedure and the analysis are automated by means of self-written software in $\mathrm{C}++$. The measured values shown are the arithmetic mean of more than one hundred single pulse events. The values take into account the measured reflectivity of the beam splitter in front of the lens wheel, the transmission through the beam splitter, and the following optics to the eye model. The measurement errors for both the incident energy and the pulse transmission are given by the standard error. The measurement uncertainties for the reflectivity of the beam splitter and for the transmission through the beam splitter and the telescope in front of the eye model are included by Gaussian error propagation. The intersection of linear fits to the horizontal and decreasing parts of the measured data is defined as LIOB threshold.

Additionally, a beam viewing camera was used to observe the focal area inside the eye model from above. If the incident pulse energy exceeds the LIOB threshold energy, laser pulses of the $5-\mathrm{kHz}$ pulse train are scattered and reflected at gas bubbles generated by previous pulses. ${ }^{23}$ This resulted in a flickering spot in the camera image visible at the location of the laser focus.

\subsection{Targets Used Inside the Eye Model and Sample Structure Preparation}

Different targets can be dipped into the chamber from above. Targets used inside the eye model were a PTFE band for closed loop AO correction and phantom structures which mimic epiretinal membranes in front of retinal tissue, which was carefully excised from porcine eyes obtained from the local slaughterhouse. For tissue sample extraction, the sclera of the porcine eyeball was scratched and pierced with a scalpel well below the vitreous base near the equatorial plane. Starting from this puncture, the eyeball was cut along the equator with surgical scissors. After complete opening of the eyeball, the vitreous body was poured out by raising the posterior eye cup inclined with forceps. A round piece of retinal tissue was excised from the posterior eye cup using a corneal trephine with an 11-mm diameter. The piece of retinal tissue was glued with cyanoacrylate on a metal bracket and dipped into the water filled chamber. A transparent synthetic foil (polyethylene, material thickness about $0.011 \mathrm{~mm}$ ) was spanned on a second bracket with a thru-hole. The second bracket is adjustable relative to the tissue sample. Figure 3 shows a schematic drawing of the sample preparation and an OCT B-mode image of an exemplary sample structure.

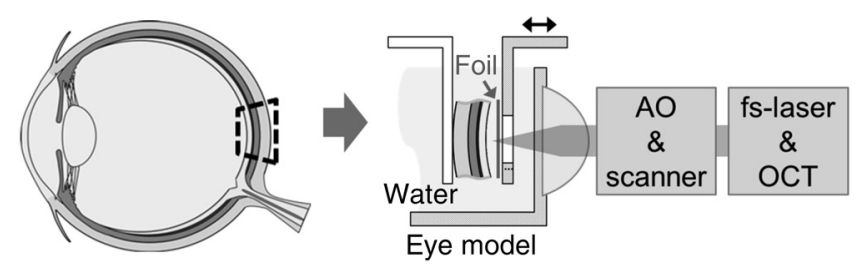

Sample structure preparation

Amplitude in $\mathrm{dB}$

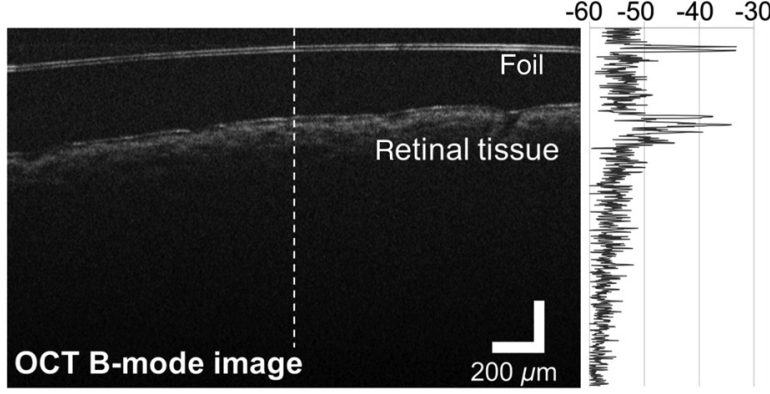

Fig. 3 Phantom structure for an epiretinal membrane: retinal tissue is carefully excised from a porcine eye and glued on a bracket which is dipped into the eye model; a synthetic foil spanned on a second bracket is adjustable in its front. The OCT B-mode image shows an exemplary sample structure. The A-scan on the right is along the dashed vertical line. 


\subsection{Parameters and Procedure for Targeted Membrane Cutting}

During the cutting experiment, the AO assisted and OCT guided fs-laser system were operated in open loop configuration (mirror surface of DM held in fixed shape). The OCT images were used to target the fs-laser focus onto the foil prior to cutting and to inspect the cutting afterward. The fs-laser focus position in the $z$ direction was determined beforehand by inserting laser cuttings into test structures [hydrated Contaflex 75 Clear (Contamac Ltd., UK) and PTFE band inside the water filled chamber] and by examining the locations of the cuttings in OCT images afterward. The phantom structure for an epiretinal membrane was then positioned inside the water filled chamber by means of linear translation stages so that the synthetic foil was at the location of the fs-laser focus in the OCT image. To cut reliably through the foil, the laser focus was shifted $\pm 10 \mu \mathrm{m}$ around the middle position in 20 steps along the $z$-axis using the DM.
For this purpose, the value of the Zernike focus coefficient was varied between each step. Per $z$-step, one line cut along the $x$-axis with a length of about 550 and $1 \mu \mathrm{m}$ in the line spot distance was performed. The line cut was performed around the center positions of the galvanometer scanners. This is close to the center of the OCT enface plane because the fs-laser and the OCT beam are superimposed in the transverse direction as accurately as possible by the shared beam path configuration and by adjustment.

\section{Results}

\subsection{Aberration Correction by Adaptive Optics}

By means of AO aberration correction, the wavefront errors of the presented setup were reduced from 270 down to $64 \mathrm{~nm}$ rms (Fig. 4, left), ignoring the Zernike terms for tilts and focus (the latter corresponding to Zernike numbers 1, 2, and 3). The laser


Fig. 4 Wavefront errors are reduced by closed loop AO correction from $270 \mathrm{~nm}$ rms in the aberrated case (top left) down to $64 \mathrm{~nm}$ rms in the corrected case (middle left), ignoring the Zernike terms for tilts and focus. The Zernike polynomial decompositions of the waveforms (bottom left) clearly show the improvement. The focus quality is enhanced after AO correction demonstrated by the PSFs calculated from the wavefront error maps (top right and middle right). The plot profiles (bottom right) are generated along the dashed lines in the PSF images. The Strehl ratio is improved from 0.11 in the aberrated case up to 0.78 in the corrected case. 


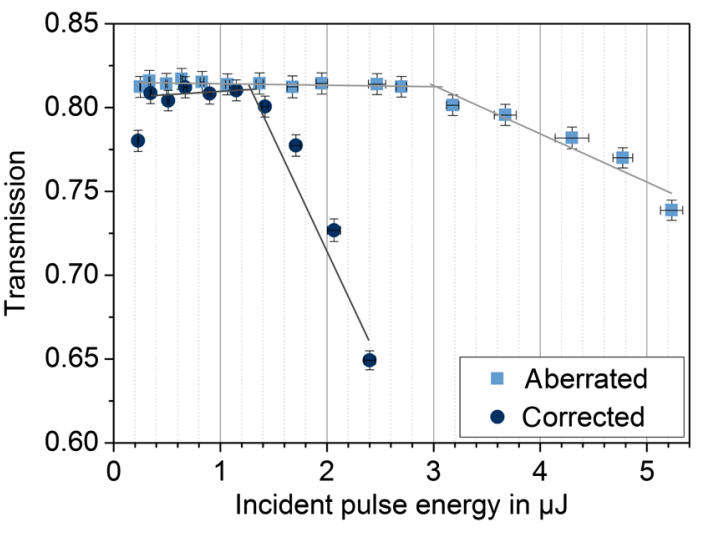

Fig. 5 The threshold energy for $\mathrm{LIOB}$ in water measured at the entrance lens of the eye model is reduced from about 3.0 down to $1.3 \mu \mathrm{J}$ pulse energy by $\mathrm{AO}$ aberration correction.

focus was improved accordingly by AO aberration correction (Fig. 4, right). The Strehl ratio, which is the ratio of the observed maximum intensity of a point source in the image plane to the theoretical maximum of a perfect optical system, was improved from 0.11 in the aberrated case up to 0.78 in the corrected case. The two-dimensional plot profiles along the dashed lines in the PSF images clearly show the improvement.

\subsection{Lowering Laser-Induced Optical Breakdown Threshold Energy by Adaptive Optics}

The improvement of the fs-laser focus resulted in increased efficiency for the cutting process. The transmission curve with increasing pulse energy bent down at much lower energy levels using $\mathrm{AO}$ for aberration correction. We deduce that the threshold energy for LIOB in water is decreased. Taking the intersection of linear fits to the nearly horizontal and decreasing parts of the curve as the LIOB threshold energy, this pulse energy fell from about 3.0 down to $1.3 \mu \mathrm{J}$ (Fig. 5). There was also a higher negative slope of transmitted energy with incident energy above the LIOB threshold energy when compensating for wavefront errors by AO. Viewing the focal area inside the eye model with a camera from above a flickering spot was observed at incident pulse energies higher than about $3.0 \mu \mathrm{J}$ in the aberrated case and higher than about $1.3 \mu \mathrm{J}$ in the corrected case.
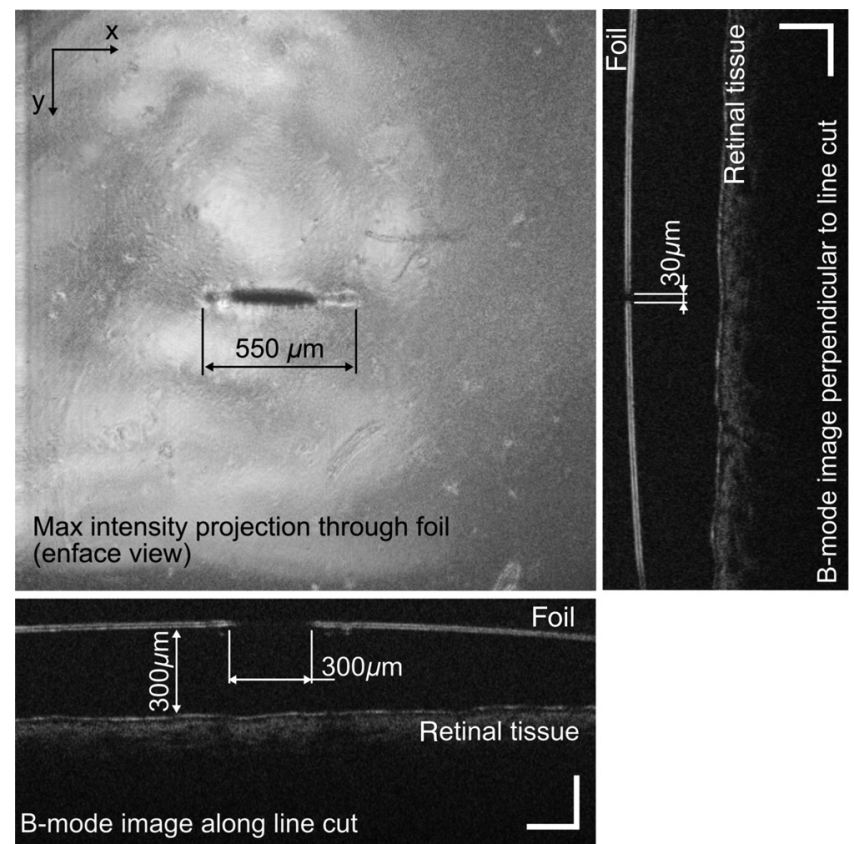

Fig. 7 Detailed views of the fs-laser line cut through the foil shown in Fig. 6. The laser cut has a length of about $550 \mu \mathrm{m}$. Over a length of about $300 \mu \mathrm{m}$, the cut is dilated perpendicular to the cutting direction. At the widest point, this dilation has a width of $30 \mu \mathrm{m}$. A laser lesion or modification of the retinal tissue behind the foil is not visible in the OCT images. The scale bars in the B-mode images indicate $200 \mu \mathrm{m}$.

\subsection{Targeting and Cutting of Phantom Structure for Epiretinal Membrane}

Targeted fs-laser cutting of a synthetic foil serving as a membrane-like phantom close to retinal tissue is shown in Fig. 6. The left picture shows a ray cast image of the OCT volume data taken immediately before fs-laser interaction; the right image shows the result after the cutting procedure. The AO system was operated in open loop in the corrected state and the used pulse energy for cutting of about $1.3 \mu \mathrm{J}$ at the entrance lens of the eye model was close to the LIOB threshold energy.

Taking a closer look at the surface of the foil in Fig. 7 shows that the foil was cut about $550 \mu \mathrm{m}$ in length. Over a length of about $300 \mu \mathrm{m}$, this cut was widened perpendicular to the cutting
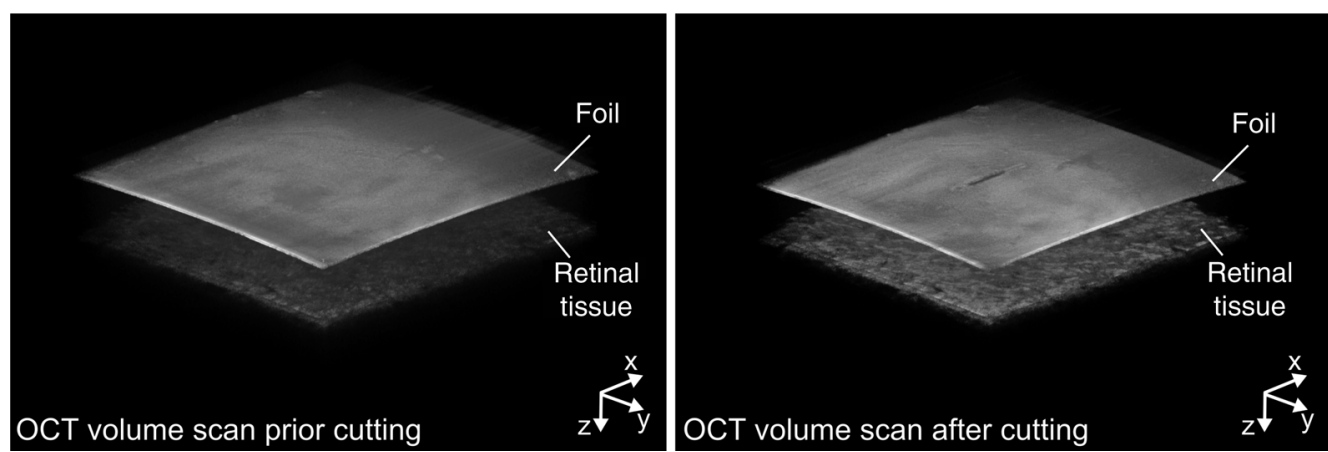

Fig. 6 Targeted fs-laser cutting of a synthetic foil close to excised retinal tissue of a porcine eye ex vivo. The AO system was operated in open loop configuration with the deformable mirror held in the corrected shape. The line cut was programmed along the $x$-axis with $550-\mu \mathrm{m}$ length. The laser pulse energy was near the LIOB threshold energy of $1.3 \mu \mathrm{J}$ at the entrance of the eye model. The OCT volumes have a lateral dimension of $2.2 \mathrm{~mm} \times 2.2 \mathrm{~mm}$. 
(a)
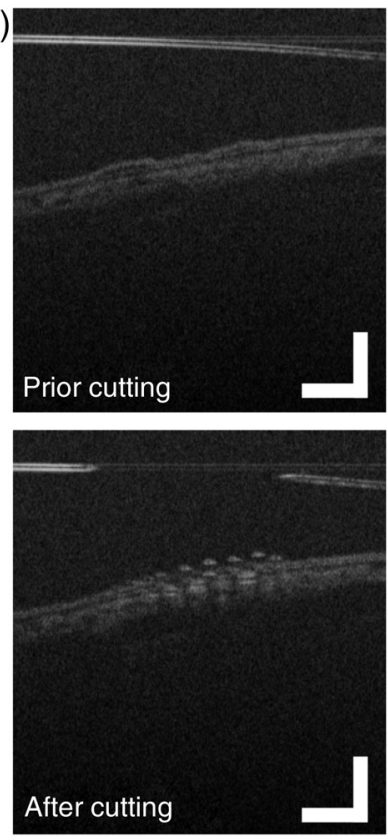

(b)
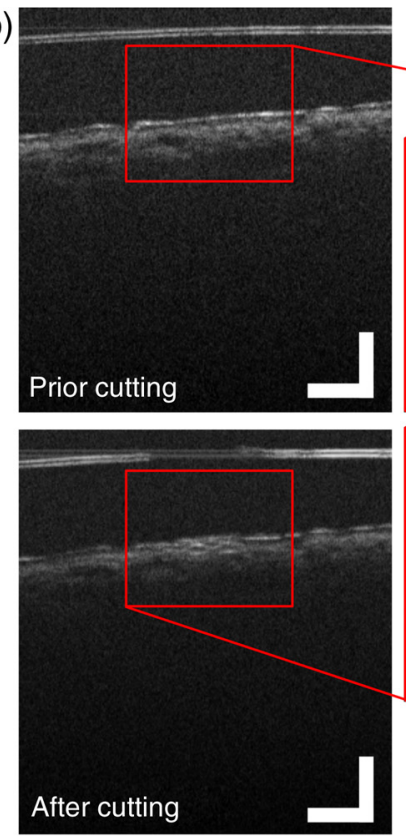
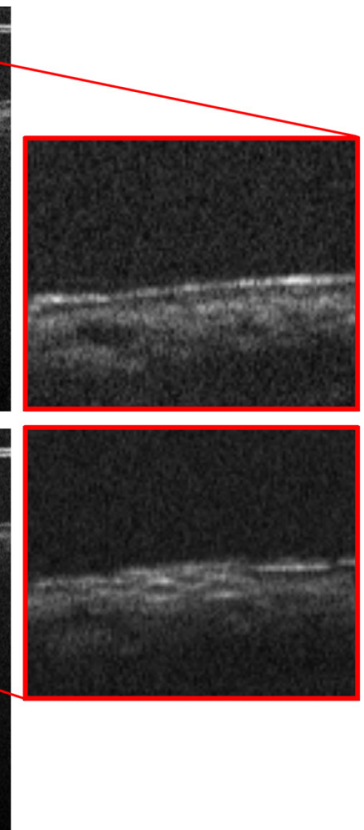

Fig. 8 Using relative high incident pulse energies for cutting lead to damages/modifications of porcine retinal tissue. (a) Severe damage of the RPE with bubble formation is observed; the system was used in the aberrated state (flat DM surface) and the pulse energy was $4.7 \mu \mathrm{J}$. (b) Slight modification is visible in the RPE after cutting; the system was used in the aberrated state and the pulse energy was $3.1 \mu \mathrm{J}$. The scale bars indicate $200 \mu \mathrm{m}$

direction, which might be due to an initial tension inside the foil by the sample preparation procedure (spanning the foil onto the bracket). At the widest point, the width of the cut was determined to be $\sim 30 \mu \mathrm{m}$ without any noticeable side fringes or laser influenced material modifications at side zones. The underlying retinal tissue was visualized by 3-D OCT volumes through the partially transparent membrane and after removing the membrane. When analyzing the individual OCT B-mode images, lesions or modifications of the retinal tissue could not be identified.

For comparison, Fig. 8 shows examples of situations in which retinal tissue was damaged or modified after cutting a foil in front of it. In Fig. 8(a), the incident pulse energy was about $4.7 \mu \mathrm{J}$. Significant damage and bubble formation are visible in the OCT B-mode image. Using incident pulse energies of about $3.1 \mu \mathrm{J}$ led to slight tissue modification in the retinal pigment epithelium (RPE) after cutting [Fig. 8(b)]. In both cases, the system was used in the aberrated state (flat DM surface).

\section{Discussion and Conclusion}

The functionality of the laboratory system for image-guided vitreoretinal fs-laser surgery was successfully demonstrated. The system integrates an $\mathrm{AO}$ subsystem with deformable mirror for spatial beam shaping and a spectrometer-based Fourier domain OCT system for laser beam guidance working properly together as a functional unit for image-guided and AO enhanced fs-laser cutting.

The AO subsystem is able to increase the energy efficiency of the cutting process when aberrations are initially present. The LIOB threshold pulse energy is lowered and the transmission after LIOB threshold decreases more steeply when correcting for aberrations. From this, it can be deduced that a safe working distance to the retina can possibly be reduced by applying AO correction. Less incident and less transmitted pulse energy generally reduces the risk of retinal damage because both the total amount of possibly transmitted energy and its absorption in the RPE are limited and the extent of collateral mechanical damage next to the focal volume is decreased. The determined lowering of the LIOB threshold pulse energy is consistent with Hansen et al. ${ }^{23}$ In that publication, a slightly different eye model was used and the LIOB threshold pulse energy was lowered from 2.3 down to $1.1 \mu \mathrm{J}$ for $5-\mathrm{kHz}$ pulse trains. The AO fslaser system described there had less complexity, but used the same AO components (DM, SHS, and software) and fslaser system. The pulse width of 250 fs measured here was slightly higher than the $120 \mathrm{fs}$ stated in Hansen et al., ${ }^{23}$ which might be due to an inferior alignment or aging of the laser system. This time using a more complex setup we measured higher wavefront errors after AO aberration correction in comparison with Hansen et al. ${ }^{23}$ (263 down to $30 \mathrm{~nm} \mathrm{rms}$ versus 270 down to $64 \mathrm{~nm}$ rms measured here). In addition to the higher system complexity, this might also be attributed to a quarter-wave plate which was used in front of the eye model in the setup described in Ref. 23. The quarter-wave plate rotates the signal polarization in the orthogonal state after double passing through the plate. Consequently, it is not clear whether the SHS in this former setup measured the wavefront of a primarily specular reflection (double pass) or the crossed polarized projection of arbitrary polarized diffuse scattering (point source single pass).

The density of free electrons at the end of an fs-laser pulse with pulse energy near the threshold is in a first approximation proportional to $I^{k}$ (multiphoton ionization, $k$ photon process). ${ }^{16}$ The generation of free electrons by cascade ionization (proportional to $I$ ) only plays a minor role for such short pulse durations. By the improvement in the Strehl ratio from 0.11 to 0.78 , one might expect a much larger reduction in the breakdown threshold than the measured reduction from 3.0 down to $1.3 \mu \mathrm{J}$. The reason why we did not measure a larger reduction in 
breakdown threshold is most likely due to the presence of local peaks (hot spots) in the focal volume of the aberrated laser beam. In the (near) diffraction-limited case only the central part of the PSF surpasses the irradiance threshold for LIOB. In the aberrated case, the irradiance distribution in the focal volume is changed and the laser pulse energy is spread over a larger region. This requires a raised total irradiance to still surpass the threshold. However, local hot spots in the irradiance can cause plasma formation that starts before the breakdown threshold is surpassed in the entire focal volume. Hot spots in the focal region were mentioned before by Vogel et al. ${ }^{19}$ to explain a discrepancy in the optical-breakdown irradiance threshold of an aberrated system when it was calculated by the use of the measured spot size compared with the threshold of an optimized system. They concluded that the presence of aberrations leads to erroneous values of the optical-breakdown threshold even when the measured spot size (instead of the diffraction-limited spot size) is used for its determination. Another reason for the smaller difference in breakdown threshold might be a stronger self-focusing due to the higher introduced pulse energy in the aberrated case.

The OCT system with its 3-D imaging capability can be used for presurgical survey of the pathology, surgical planning, intrasurgical positioning of the fs-laser focus, and for monitoring cuts leading to segmentation of target structures. The clinical functionality of such surgical guidance is proved by systems for fs-laser assisted cataract surgery with integrated OCT imaging. ${ }^{5}$ The shared path configuration of OCT and fs-laser beam as described here has the advantage of requiring fewer optomechanics and costly components, e.g., additional scanners, can be spared. Real-time monitoring of the cutting process and feedback loop control of the laser cutting parameters, on the other hand, are difficult to implement in such a shared path approach. A laser source would be required which can provide pulses on demand, so that laser pulses could be applied whenever the scanners are at the right positions in the OCT images. An alternative system design would be a modular OCT system with a scanner working independently from the laser scanner. Using this modular approach, the sites can be visualized in real-time during the entire process including the ongoing laser surgery. Here, beam combining optics and the cross reference of OCT coordinate system and laser coordinate system are more demanding.

In our shared path system, AO was used to reshape both the focus of OCT and fs-laser. In Ref. 32, we demonstrated that the OCT lateral resolution could be slightly improved from 9 to $7 \mu \mathrm{m}$ after reducing wavefront errors by AO aberration correction using fs-laser pulses reflected at a PTFE band inside the eye model as a point source. This minor impact of AO aberration correction on image quality of our parallel OCT system might be due to noncommon path aberrations introduced by the beam expander in the OCT beam path and the telescope between the DM and the SHS (refer to Fig. 2). The impact on image quality may increase when reducing or avoiding these noncommon path aberrations. Another explanation for why AO has a minor effect on the OCT image quality might be that the OCT beam is underfilling the DM. The diameter of the OCT beam at the DM is $\sim 0.86$ times the beam diameter of the fs-laser which is filling the DM. The alternative modular system approach would take away the possible advantage of simultaneously reshaping the OCT focus to the laser focus. However, Shemonski et al. ${ }^{33}$ published a fully automated computational approach that enables high-resolution in vivo ophthalmic imaging without the need for hardware-based AO, so that the beam of the modular OCT system does not have to pass a wavefront modulator for aberration correction.

The tissue sample preparation is a delicate and demanding task. When pouring out the vitreous body, the photoreceptor layer can easily peel off from the posterior eye cup. In this respect, the use of the corneal trephine to excise a piece of retinal tissue is even more critical. The tissue samples shown in the cutting experiments are lacking the neural layer of the retina. However, the RPE was retained on the choroid. Because the RPE is a highly pigmented layer of the retina, we expect the strongest absorption of the laser light in this layer. Therefore, lesions or modifications by absorbed laser light should appear first in the RPE.

Challenging for a clinical translation will be the online determination of the LIOB threshold energy. In case of an LIOB, part of the incident energy is absorbed during plasma generation or is reflected or scattered at the plasma. Therefore, one possibility to determine the LIOB threshold energy in situ could be the detection of back reflected energy. The measuring setup could consist of a beam splitter and a detector pair in the detection arms introduced by the beam splitter as described by Nahen and Vogel. ${ }^{34}$ One detector measures the pulse energy in reflection of the beam splitter for the incoming light and one detector measures the pulse energy in reflection of the beam splitter for the outgoing light. As an alternative method, LIOB threshold energy can possibly be determined by means of a modular and simultaneously detecting OCT system.

Our presented laboratory system is an extension of the AOFL instrument given in Hansen et al. ${ }^{23}$ to a lab demonstrator of a seeing laser scalpel. The system integrates the function and synchronized interplay of a scanning unit for focus positioning and a spectrometer-based Fourier domain OCT system for laser beam guidance. Fine tuning of the focus position in the $z$-direction is possible by means of the DM. Focus positioning over a longer $z$-range can be realized by implementing the last telescope as a Badal optometer, by replacing the lens wheel by a focus tunable lens or by both. Two key problems for vitreoretinal fs-laser surgery were addressed: aberrations and focus positioning. The laboratory system was used for targeted cutting of a synthetic foil close to excised porcine retinal tissue inside an eye model. This phantom mimics the typical geometrical situation of an epiretinal membrane. Laser lesions or modifications in the retinal tissue were not visible in the OCT images for the presented epiretinal cut using the aberration corrected system and pulse energies near the LIOB threshold energy. Although the use of OCT to detect RPE damage is questionable, because RPE cells may be damaged without producing observable OCT lesions, OCT survey gives a good first indication for the possibility of cutting close to the retina without side effects. To ensure that the laser procedure is really safe and to determine exact values at which distance to the retina laser cutting can be performed without causing damage, further studies are required. Possible methods of analysis are, e.g., histopathological examinations, scanning electron microscopy, assays for cell viability using a viability stain and fluorescence microscopy or neurophysiological studies in an animal model. Although in situ tissue optical properties may differ from this setting with an artificial membrane before porcine retina, the principal advantage of focus positioning and threshold lowering will hold true for an epiretinal membrane. Moreover, the laboratory system can now 
be used for further feasibility tests with more realistic phantoms reaching up to ex vivo porcine eyeballs with more demanding aberrations.

\section{Disclosures}

The authors have no financial interests in the manuscript and no other potential conflicts of interest to disclose.

\section{Acknowledgments}

This work was part of the research project IKARUS (innovative cataract, age-related presbyopia, and retina treatment with ultrashort pulsed lasers; No. 13N11847) and was sponsored by the German Federal Ministry of Education and Research (BMBF). The authors thank Konstanze Horke for helpful discussions and assistance regarding the preparation of the ocular tissue and Gesche Knoop and Timo Gewohn for helpful assistance in the laboratory.

\section{References}

1. T. Juhasz et al., "Corneal refractive surgery with femtosecond lasers," IEEE J. Quantum Electron. 5(4), 902-910 (1999).

2. G. Sutton, M. Lawless, and C. Hodge, "Laser in situ keratomileusis in 2012: a review," Clin. Exp. Optometry 97(1), 18-29 (2014).

3. T. Ripken et al., "fs-laser induced elasticity changes to improve presbyopic lens accommodation," Graefes Arch. Clin. Exp. Ophthalmol. 246(6), 897-906 (2008).

4. S. Schumacher et al., "Femtosecond laser induced flexibility change of human donor lenses," Vision Res. 49(14), 1853-1859 (2009).

5. D. V. Palanker et al., "Femtosecond laser-assisted cataract surgery with integrated optical coherence tomography," Sci. Transl. Med. 2(58), 58ra85 (2010)

6. K. E. Donaldson et al., "Femtosecond laser-assisted cataract surgery," J. Cataract. Refract. Surg. 39(11), 1753-1763 (2013).

7. J. Bottós et al., "Vitreomacular traction syndrome," J. Ophthalmic Vision Res. 7(2), 148-161 (2012).

8. Y. Barak, M. A. Ihnen, and S. Schaal, "Spectral domain optical coherence tomography in the diagnosis and management of vitreoretinal interface pathologies," J. Opthalmol. 2012, 876472 (2012).

9. D. H. W. Steel and A. J. Lotery, "Idiopathic vitreomacular traction and macular hole: a comprehensive review of pathophysiology, diagnosis, and treatment," Eye 27, S1-S21 (2013).

10. A. Koizumi et al., "Three-dimensional evaluation of vitreomacular traction and epiretinal membrane using spectral-domain optical coherence tomography," Am. J. Ophthalmol. 145(3), 509-517 (2008).

11. J. Sebag and M. Y. Wang, "Combined spectral-domain optical coherence tomography/scanning laser ophthalmoscopy imaging of vitreous and the vitreo-retinal interface," in Medical Retina-Focus on Retinal Imaging, F. G. Holz and R. F. Spaide, Eds., pp. 156-168, SpringerVerlag, Berlin, Heidelberg (2010).

12. F. Koch, "Netzhaut- und Glaskörperchirugie," in Augenheilkunde, 2nd ed., A. J. Augustin, Ed., pp. 741-775, Springer-Verlag, Berlin, Heidelberg (2001).

13. C. A. Rennie et al., "Nd:YAG laser treatment for premacular subhyaloid haemorrhage," Eye 15(4), 519-524 (2001).

14. L. Znaor et al., "OCT-guided Nd:YAG laser hyaloidotomy," Spektrum Augenheilkd. 23(6), 423-426 (2009).

15. A. Vogel, "Nonlinear absorption: intraocular microsurgery and laser lithotripsy," Phys. Med. Biol. 42, 895-912 (1997).

16. A. Vogel et al., "Mechanisms of femtosecond laser nanosurgery of cells and tissues," Appl. Phys. B 81(8), 1015-1047 (2005).

17. A. Heisterkamp et al., "Optimierung der Laserparameter für die intrastromale Schnittführung mittels ultrakurzer Laserpulse," Ophthalmologe 98(7), 623-628 (2001).

18. T. O. Salmon and C. van de Pol, "Normal-eye Zernike coefficients and root-mean-square wavefront errors," J. Cataract. Refract. Surg. 32(12), 2064-2074 (2006).
19. A. Vogel et al., "Influence of optical aberrations on laser-induced plasma formation in water and their consequences for intraocular photodisruption," Appl. Opt. 38(16), 3636-3643 (1999).

20. J. Liang, D. R. Williams, and D. T. Miller, "Supernormal vision and high-resolution retinal imaging through adaptive optics," J. Opt. Soc. Am. A 14(11), 2884-2892 (1997).

21. A. Roorda et al., "Adaptive optics scanning laser ophthalmoscopy," Opt. Express 10(9), 405-412 (2002).

22. B. Hermann et al., "Adaptive-optics ultrahigh-resolution optical coherence tomography," Opt. Lett. 29(18), 2142-2144 (2004).

23. A. Hansen et al., "Lowered threshold energy for femtosecond laser induced optical breakdown in a water based eye model by aberration correction with adaptive optics," Biomed. Opt. Express 4(6), 852-867 (2013).

24. A. Krüger et al., "Towards femtosecond laser surgery guidance in the posterior eye: utilization of optical coherence tomography and adaptive optics for focus positioning and shaping," Proc. SPIE 8935, 89350L (2014).

25. P. Hahn et al., "Intrasurgical human retinal imaging with manual instrument tracking using a microscope-integrated spectral-domain optical coherence tomography device," Transl. Vision Sci. Technol. 4(4), 1 (2015).

26. J. P. Ehlers et al., "Utility of intraoperative optical coherence tomography during vitrectomy surgery for vitreomacular traction syndrome," Retina 34(7), 1341-1346 (2014).

27. E. J. Chung et al., "OCT-guided hyaloid release for vitreomacular traction syndrome," Korean J. Ophthalmol. 22(3), 169-173 (2008).

28. L.-O. Hattenbach et al., "Intraoperative Echtzeit-OCT in der Makularchirurgie," Ophthalmologe 113(8), 656-662 (2016).

29. J. C. Wyant and K. Creath, "Basic wavefront aberration theory for optical metrology," in Applied Optics and Optical Engineering, Vol. 11, R. R. Shannon and J. C. Wyant, pp. 1-54, Academic Press, San Diego, California (1992).

30. F. Köttig et al., "An advanced algorithm for dispersion encoded full range frequency domain optical coherence tomography," Opt. Express 20(22), 24925-24948 (2012).

31. A. Roorda, D. T. Miller, and J. Christou, "Strategies for high-resolution retinal imaging," in Adaptive Optics for Vision Science: Principles, Practices, Design, and Applications, J. Porter et al., Eds., pp. 235287, John Wiley \& Sons, Hoboken, New Jersey (2006).

32. B. Matthias et al., "Concept for image-guided vitreo-retinal fs-laser surgery: adaptive optics and optical coherence tomography for laser beam shaping and positioning," Proc. SPIE 9307, 93070Z (2015).

33. N. D. Shemonski et al., "Computational high-resolution optical imaging of the living human retina," Nat. Photonics 9, 440-443 (2015).

34. K. Nahen and A. Vogel, "Plasma formation in water by picosecond and nanosecond Nd:YAG laser pulses-part II: transmission, scattering, and reflection," IEEE J. Quantum Electron. 2(4), 861-871 (1996).

Ben Matthias graduated in technical physics at Leibniz Universität Hannover in 2013. Since 2010, he has been working at the Laser Zentrum Hannover e.V. in the Biomedical Optics Department. His research is focused on image-guided fs-laser surgery in the field of ophthalmic technologies taking advantage of optical coherence tomography and adaptive optics.

Miroslav Zabic graduated in technical physics at Leibniz Universität Hannover in 2015. After finishing his master's thesis at the Laser Zentrum Hannover e.V. in the Biomedical Optics Department, he started to work in the field of hyperspectral imaging at Cubert $\mathrm{GmbH}$.

Dorothee Brockmann graduated from the Hannover Medical School in 1993. She started special ophthalmological training at the Hannover Medical School in 1993 and finished specialization in 1998. She has been working at the eye clinic at Hannover Medical School since 1993, since 1998 as senior registrar, and several years as deputy to the head of the department. Her special subjects are vitreoretinal surgery and pediatric ophthalmology. 
Alexander Krüger graduated in physics in 1998 and finished his dissertation about optical parametric oscillators in January 2003 at the University of Bonn. Optical coherence tomography became one of his major interests in the years 2003 to 2007 at the Medical Faculty of Technical University Dresden. Since 2008, he has worked at the Laser Zentrum Hannover e.V., and now he is head of the Image-Guided Laser Surgery Group in the Biomedical Optics Department.
Tammo Ripken studied physics with diploma and received his PhD from Leibniz Universität Hannover in 2007. Since 2001, he has been working at the Laser Zentrum Hannover e.V., from 2005 to 2008 as a project leader for fs-laser in ophthalmology, and from 2008 to 2010 as the head of Laser Medicine Group. Since 2011, he has been acting as the head of Biomedical Optics Department. His main research areas are laser-assisted medicine, laser-tissue interaction, and laser-based medical imaging. 